\title{
Impact of pasteurization on the antibacterial properties of human milk
}

\author{
Marjan Van Gysel • Veerle Cossey • Steffen Fieuws • \\ Annette Schuermans
}

Received: 21 December 2011 /Revised: 18 April 2012 / Accepted: 27 April 2012 /Published online: 12 May 2012

(C) Springer-Verlag 2012

\begin{abstract}
Growing evidence favours the use of human milk for the feeding of preterm newborns based on its many beneficial effects. Despite the many benefits, human milk has been associated as a possible vehicle of transmission for a number of infections. Although pasteurization of human milk can diminish the risk of neonatal infection, it also significantly reduces the concentrations of immunological components in human milk due to thermal damage. In order to evaluate the impact of pasteurization on the antibacterial properties of human milk, we aimed to compare the capacity of raw and pasteurized human milk to inhibit bacterial proliferation. Therefore, a single milk sample was collected from ten healthy lactating mothers. Each sample was divided into two aliquots; one aliquot was pasteurized, while the other was kept raw. Both aliquots were inoculated either with Escherichia coli or Staphylococcus aureus and incubated at $37^{\circ} \mathrm{C}$ during $8 \mathrm{~h}$. Viable colony counts from the inoculated samples were performed at regular time points to compare the bacterial growth in both forms of breast milk. Relative to the tryptic
\end{abstract}

\footnotetext{
M. Van Gysel

Laboratory for Microbiology, Regional Hospital Sint-Maria, Halle, Belgium

V. Cossey $(\bowtie) \cdot$ A. Schuermans

Department of Hospital Hygiene and Infection Control, University Hospitals Leuven,

Leuven, Belgium

e-mail: veerle.cossey@uz.kuleuven.ac.be

V. Cossey

Neonatal Intensive Care Unit, University Hospitals Leuven, Leuven, Belgium

S. Fieuws

Department of Public Health, Interuniversity Centre for

Biostatistics and Statistical Bioinformatics,

Leuven, Belgium
}

soy broth control sample, both raw and pasteurized milk samples exhibited an inhibitory effect on the growth of $E$. coli and $S$. aureus. Compared with the raw portion, growth inhibition was significantly lower in the pasteurized milk at every time point beyond T0 (after 2, 4 and $8 \mathrm{~h}$ of incubation) ( $p=$ 0.0003 for E. coli and $p<0.0001$ for $S$. aureus). Conclusion: Our study shows that pasteurization adversely affects the antibacterial properties of human milk.

Keywords Pasteurization · Human milk · Immunological components $\cdot$ Neonatal infection

\section{Introduction}

Several studies reported the beneficial effects of human milk for full-term as well as for premature infants $[3,40]$. The benefits of mother's own milk go beyond provision of a balanced source of nutrients that meet the dietary requirements of the infant. It also contains bioactive factors that protect the infant from illness through a variety of mechanisms, including stimulating immune function development, anti-inflammatory and antimicrobial activity and enhancing growth and development of tissues [7,24,33].

Convincing data from both high- and low-income countries provide strong evidence that feeding on raw mother's milk offers protection against a wide range of infectious diseases, including otitis media, diarrhoea, necrotizing enterocolitis (NEC) and respiratory tract infections $[11,19]$. The evidence for the benefits of pasteurized donor milk is limited, but it has been shown that feeding with donor milk lowers the risk of developing NEC in preterm infants compared to formula milk [32].

Human milk may, however, also be a vehicle for microorganisms derived from the mother or the environment 
during collection, storage and handling of the milk. Between 60 and $80 \%$ of samples of expressed human milk are contaminated with high amounts of skin contaminants, including coagulase-negative staphylococci (CoNS), alphahaemolytic streptococci and diphteroids $[23,28]$, or pathogens such as cytomegalovirus or Escherichia coli [36], Serratia marcescens [13], Pseudomonas aeruginosa [14] or Klebsiella species [10].

As a safeguard against the transmission of viral and bacterial pathogens, best practice requires that donated human milk must undergo Holder pasteurization prior to being fed to recipients $[15,18,26,40]$. Although pasteurization assures the microbiological safety of human milk, the mechanism of thermal inactivation of bacteria is detrimental to the bioactivity of the milk, since most of the proteins will denature when exposed to heat. Several studies have shown that Holder pasteurization reduces to some extent the activity of important immunomodulating components $[4,8,21,38]$. Hence, thermal treatment may not only impair the beneficial antibacterial properties of human milk, but may also increase its susceptibility to subsequent bacterial contamination.

We undertook the current study to evaluate the effect of Holder pasteurization on the capability of human milk to inhibit bacterial proliferation. Furthermore, we wanted to examine the difference in antimicrobial capacity between human milk collected from mothers who delivered prematurely and that collected from mothers who delivered at term.

\section{Materials and methods}

\section{Sample source}

Ten human milk samples were collected from ten healthy lactating women whose babies were admitted to the neonatal intensive care unit of the University Hospitals of Leuven. The women did not show signs of mastitis nor did they receive antibiotics in the week before sample collection. Each sample (minimal $30 \mathrm{~mL}$ ) was collected in one session using an electric breast pump (Ameda Breastfeeding Products, IL, USA). To ensure the optimum quality of milk, each donor received oral and written instructions concerning hygiene, collection, storage and transport when expressing for her own infant. Nipples and areolas were cleaned with water before expression. The first milk was not discarded. Stage of lactation, birth weight and gestational age were recorded. Each human milk sample was divided into two aliquots; one aliquot was kept raw, while the other was to be pasteurized. Both aliquots were stored immediately under refrigerated conditions $\left(2-5{ }^{\circ} \mathrm{C}\right)$ and processed within a median of $22 \mathrm{~h}$ (and a maximum of $48 \mathrm{~h}$ ) after expression, mimicking the feeding practices in the NICU. The time elapsed from expression to the start of the microbiological assay was less than $48 \mathrm{~h}$ for all milk samples.

Prior to sampling, all donors gave their informed consent to participate in this study. The study protocol was approved by the local Ethics Advisory Committee of the University Hospitals of Leuven.

Thermal treatment

For pasteurization of the samples, the Holder process $\left(62.5^{\circ} \mathrm{C}\right.$ for $\left.30 \mathrm{~min}\right)$ was applied using a S75 TES pasteurizer (Sterifeed, UK).

Bacterial proliferation assay

From each donor, we received a pasteurized and raw portion of milk; each portion was divided into two aliquots (Fig. 1). One aliquot was inoculated with Staphylococcus aureus (S. aureus ATCC 6538) and the other with E. coli NCTC 10538. For preparation of the inoculum, the $S$. aureus and E. coli strain were cultured overnight on tryptic soy agar (Oxoid) in order to obtain fresh colonies, suspended in sterile normal saline $(0.9 \% \mathrm{NaCl})$, which was adjusted to a turbidity equal to that of a McFarland no. 1 standard.

Of each bacterial suspension, $250 \mu \mathrm{L}$ was separately added to $4.75 \mathrm{~mL}$ of human milk (both to the raw and pasteurized portion) and to $4.75 \mathrm{~mL}$ of tryptic soy broth (TSB, Oxoid), the latter acting as a normal growth control. After inoculation of $100 \mu \mathrm{L}$, the samples and TSB were immediately incubated at $37{ }^{\circ} \mathrm{C}$ on a mechanical agitator (200 rpm).

During incubation, viable colony counts were carried out at regular time points $(0,4$ and $8 \mathrm{~h})$ by culturing appropriate dilutions of the inoculated milk and control samples on MacConkey agar (Oxoid) for E. coli and on SAID agar (BioMérieux) for $S$. aureus at $37^{\circ} \mathrm{C}$ for 24 and $48 \mathrm{~h}$, respectively. The unspiked milk samples were also inoculated on appropriate media to check for sterility in the pasteurized portion and for background level of bacteria in the raw portion. Colony counts were performed in triplicate and expressed as viable colony-forming units (cfu) per millilitre of milk. The degree of growth inhibition in percent was calculated as the relative difference in $E$. coli or $S$. aureus counts between control and milk samples.

Statistical analysis

The evolution in growth inhibition was compared between raw and pasteurized milk using a linear mixed model for repeated measures for E. coli and S. aureus separately. A direct likelihood approach was adopted using an unstructured covariance matrix for the four 


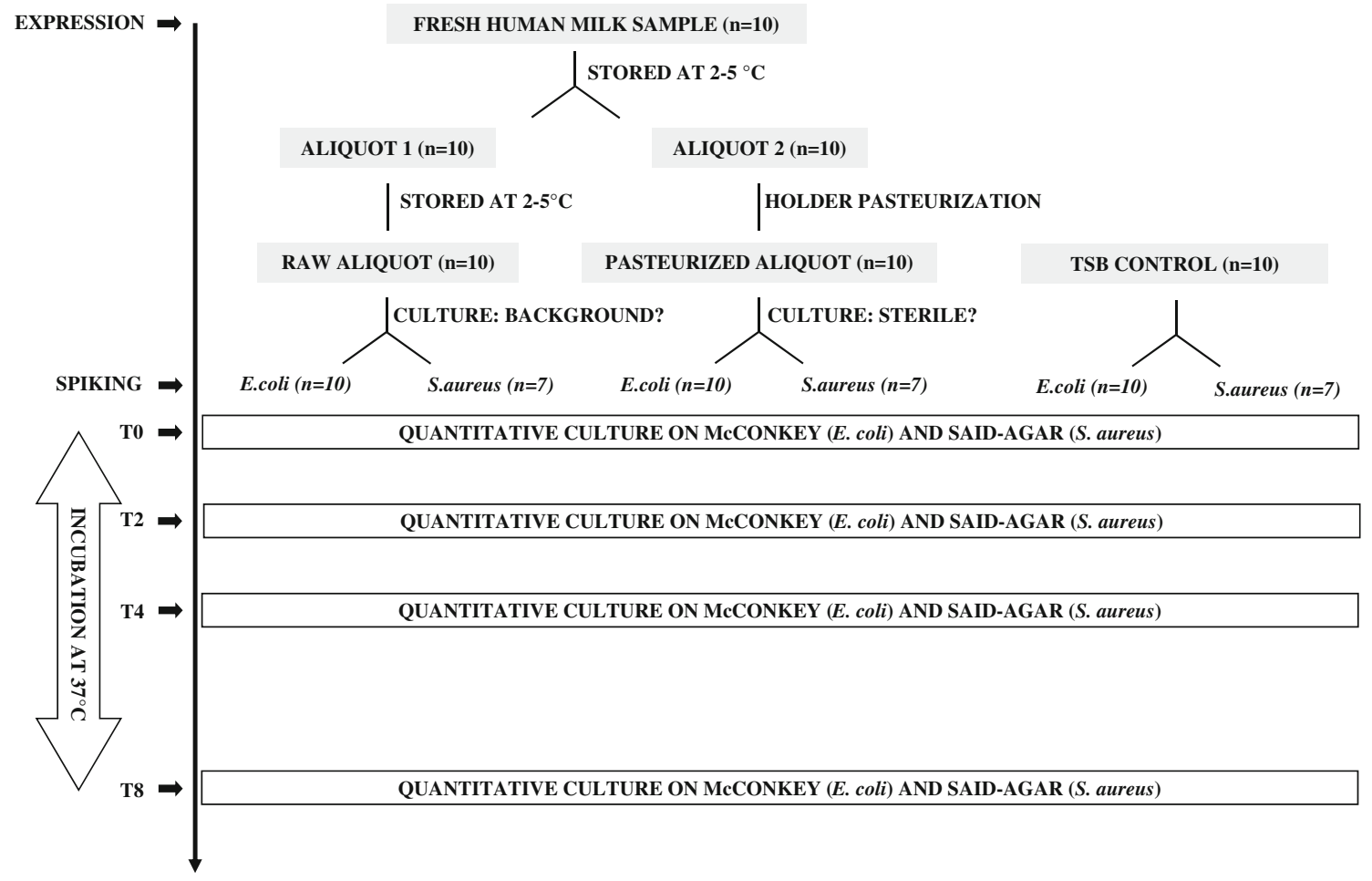

Fig. 1 Schematic overview of sample processing

repeated measures in time per specimen-type of milk combination; a random effect of specimen per point in time was added to allow correlation at a specific moment between the inhibition observed for raw and pasteurized milk. Based on this model, comparisons between both types of milk were made for the inhibition at each of the four time points as well as for the changes (versus T0) in inhibition at $\mathrm{T} 2, \mathrm{~T} 4$ and $\mathrm{T} 8$. Figures were created with least-squares means and $95 \%$ confidence intervals. Due to the low number of specimens, it was assumed that the $8 \times 8$ covariance matrix of the repeated measures could be described by a lower number of parameters and that the assumption of normality was fulfilled. Therefore, as a sensitivity analysis, levels of inhibition were compared between both types of milk using signed rank tests at each point in time. Further, the model of repeated measures has been extended with the effect of term versus preterm. As such it was evaluated (a) if the difference in evolution of inhibition between pasteurized and raw milk depends on the moment of delivery, (b) if the difference in inhibition between pasteurized and raw milk depends on the moment of delivery and (c) if the evolution of inhibition differs between term and preterm milk. For all analyses, $p$ values smaller than 0.05 were considered significant. All analyses were performed using SAS software, version 9.2, of the SAS System for Windows (copyright (C) 2002 SAS Institute Inc., Cary, NC, USA).

\section{Results}

A total of ten unfortified human milk samples were analysed. Five samples were collected from mothers who delivered prematurely at a median gestation of 29 weeks (range, 26-30 weeks) and whose babies had a median birth weight of 1,315 $\mathrm{g}$ (range, 880-1,345 g). The other five samples were obtained from mothers who delivered at term at a median gestation of 37 weeks (range, 36-38 weeks); their babies had a median birth weight of 3,620 $\mathrm{g}$ (range, $1,735-4,300 \mathrm{~g})$. According to the stage of lactation, all samples consisted of mature milk with collection around the 25th day of postnatal age (IQR, 21-37 days). In order to check for contamination and to investigate the bacterial background level, we cultured all breast milk samples before spiking and found that all pasteurized portions were sterile, while all raw portions were predominantly contaminated with CoNS. Additional microorganisms identified in some of the samples included alpha-haemolytic and nonhaemolytic streptococci and diphteroids, and in one sample, we detected Acinetobacter lwoffii. The total bacterial count was $\leq 10^{5}$ cfu per millilitre of milk in all samples, in accordance with the applied limits for donor milk in milk banks [27]. In three samples, we cultured S. aureus, and those were excluded from the data analysis of the $S$. aureus proliferation assay, because it was not possible to distinguish between the endogenous and the added strain in our experiment. At all time points beyond $\mathrm{T} 0$, we observed that 
E. coli and S. aureus grew significantly less in the milk samples (in raw as well as in pasteurized) than in the TSBcontrol sample $(p<0.05)$.

The main finding of our experiment was a significantly higher growth inhibition of both E. coli and $S$. aureus in the unpasteurized milk compared to the corresponding pasteurized portion ( $p=0.0003$ for E. coli, $p<0.0001$ for $S$. aureus). The observed difference was significant at each of the three time points beyond T0 (after 2, 4 and $8 \mathrm{~h}$ of incubation).

For both bacteria types, there was a significant increase in growth inhibition over time in the pasteurized milk $(p<0.0001$ for E. coli and S. aureus $)$ as well as in the raw milk ( $p<0.0001$ for both species). The evolution of the growth inhibition observed for both forms of milk during incubation is illustrated in Figs. 2 and 3. When comparing the $E$. coli and $S$. aureus assay, we found that the difference in growth inhibition between pasteurized and unpasteurized milk did not depend on the bacterial species $(p=0.81)$.

Additionally, our data showed that the difference in growth inhibition between pasteurized and raw milk did not depend on whether the milk was collected from preterm or from term mothers $(p=0.53$ for $E$. coli and $p=0.11$ for $S$. aureus). Furthermore, we found that, after 4 and $8 \mathrm{~h}$ of incubation, milk from mothers who delivered at term had a significantly higher inhibitory effect on the growth of $E$. coli than milk from preterm mothers $(p=0.02$ at $4 \mathrm{~h}$ and $p=0.006$ at $8 \mathrm{~h}$ ), as shown in Fig. 4. For $S$. aureus, we did not find a significant difference in growth inhibition between term and preterm breast milk at any time point beyond T0 $(p=0.41$ at $2 \mathrm{~h}, p=0.22$ at $4 \mathrm{~h}$ and $p=0.21$ at $8 \mathrm{~h}$ of incubation).

\section{Discussion}

Human milk is the golden standard of enteral nutrition for all newborns, including preterm infants, because of its unique nutritional and immunological characteristics [11].

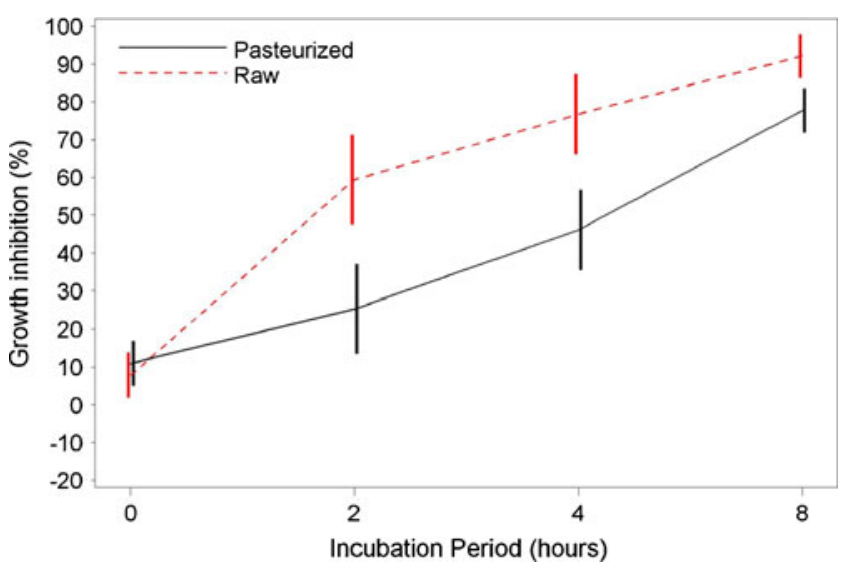

Fig. 2 Evolution of growth inhibition of E. coli during incubation. Means and $95 \%$ confidence intervals are plotted

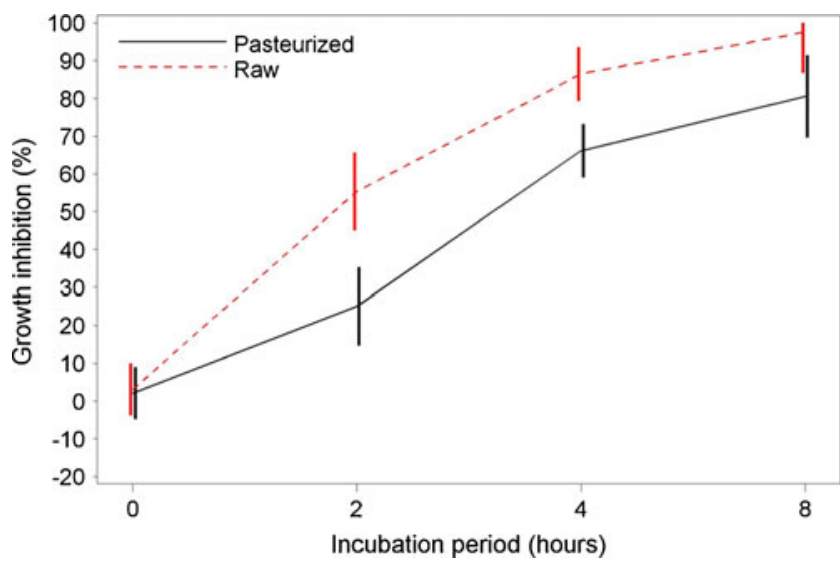

Fig. 3 Evolution of growth inhibition of $S$. aureus during incubation. Means and $95 \%$ confidence intervals are plotted

The immunomodulatory properties of fresh mother's own milk have been widely studied. It is clear that protein as well as nonprotein constituents contribute to the natural immune defence provided by breastfeeding, through a variety of ways, including direct inhibition of pathogens, neutralization of bacterial toxins, sequestration of bacterial nutrients as iron, modification of intestinal microflora and stimulation of the mucosal immune system. Examples of important vertically transferred anti-infective components include oligosaccharides, leucocytes, secretory IgA, complement factors, cytokines, lactoferrin and lysozyme $[7,12,20]$.

These anti-infective components of human milk may be extremely valuable for preterm infants. They are particularly susceptible to infection as they do not benefit from the transplacental transfer of maternal immunoglobulins, which occurs primarily after 32 weeks of gestation. Furthermore, various studies reported higher concentrations of immune factors in colostrum produced by mothers of preterm newborns than in colostrum of term mothers, suggesting a natural adaptation of the milk content to compensate for the extreme immaturity of the immune system of preterm babies

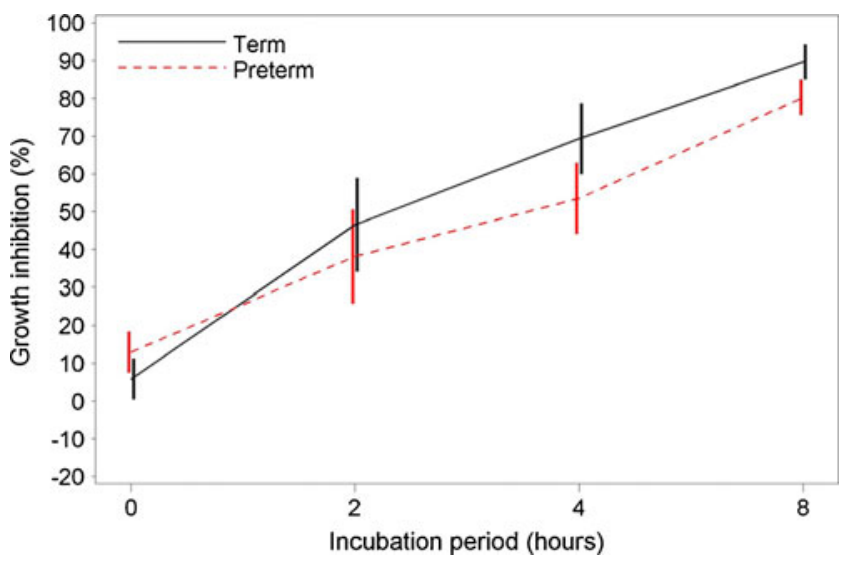

Fig. 4 Comparison of growth inhibition of $E$.coli between preterm and term milk. Means and $95 \%$ confidence intervals are plotted 
$[2,17]$. Moreover, substantive evidence exists that mother's own milk is contributory in reducing morbidity in very low birth weight infants [25,34].

Yet, during the early neonatal period, the neurological immaturity and respiratory distress of preterm infants prevent them from sucking at their mothers' breast, and they are initially fed human milk through a nasogastric tube. Given the well-documented hazards of bacterial contamination of expressed human milk and considering the frequent excretion of cytomegalovirus during lactation, mother's own milk is pasteurized before use at the University Hospitals of Leuven $[13,14,22,36]$. The pasteurization process may not be ideal for human milk, and this practice remains a controversial issue in NICUs worldwide. Inevitably, the mechanism of thermal inactivation of bacteria results in a considerable loss of beneficial proteins present in human milk. Although several studies already reported reduced levels of lysozyme [1,38], secretory IgA, lactoferrin, lactoperoxidase [1] and sCD14 [8] after Holder pasteurization, we conducted this study in order to find out to what extent the global antibacterial capacity of human milk is preserved following pasteurization. Complementary to the study conducted by Silvestre et. al., who documented that unfortified fresh human milk presents inhibitory activity on the growth of $E$. coli that is partly lost after pasteurization [35], we investigated the effect of pasteurization on the antibacterial properties of breast milk towards a Gram-positive as well as a Gram-negative species. E. coli and S. aureus strains were chosen on the grounds of being common pathogens causing contamination of milk and neonatal infections [5,37,39]. It is of interest that, according to our study design, milk samples had not been frozen prior to processing and pasteurized and raw samples were paired, which means that they originated from the same donor. Consistent with the findings of other authors $[1,35]$, our data show that untreated human milk mutes the proliferation of both $E$. coli and $S$. aureus and that, after thermal treatment, the inhibitory effect of human milk is partly lost. The present study also demonstrates that pasteurization of human milk impairs its inhibitory activity on $E$. coli to the same extent as on $S$. aureus and that the inhibitory activity of human milk on both bacterial species increases during incubation.

From this study, we conclude that, relative to a TSBcontrol, pasteurized milk still provides substantial inhibition of the growth of E. coli and $S$. aureus, albeit significantly reduced compared to untreated milk. This loss of antibacterial capacity can partially be explained by the reduced concentrations and activities of major immunologic components with both specific and non-specific bactericidal and bacteriostatic activities due to thermal damage [35]. Another possible contributing factor to the enhanced antibacterial activity of raw human milk is the presence of commensal bacteria that are thought to inhibit pathogenic growth by their production of bacteriocins and by competition for nutrients [16].

The observed increase in inhibitory effect during incubation is hypothesized to be partially due to a progressive increase in free fatty acids by the effect of milk lipases. Free fatty acids are known to have very potent cytolytic effects on microorganisms [30]. Additionally, the enhancement of antibacterial activity during incubation might be attributed to the involvement of complement which is characterized by a certain delay from activation to onset of bacterial killing $[20,29]$.

The secondary goal of our experiment was to compare the antibacterial properties of preterm and term breast milk in vitro. We did not find a significant difference in growth inhibition of $S$. aureus between preterm and term milk. Yet, contrary to what we expect based on the higher concentrations of immune factors reported in preterm milk, we found that term milk was more inhibitory to the growth of $E$. coli than preterm milk after 4 and $8 \mathrm{~h}$ of incubation. Although this finding should be interpreted with caution because of the small sample size, it does agree with the observations described by Castellote et al. [6]. They stated that the adaptation, responsible for higher concentrations of bioactive factors in colostrum of mothers who delivered between 30 and 37 weeks of gestation, is not efficient when delivery occurs very prematurely (before 30 weeks). In addition, they found that, irrespective of the weeks of gestation, the adaptation seems transient, since no differences in immune composition could be found in mature milk.

Overall, our data demonstrate a reduced capability of pasteurized human milk to curb bacterial proliferation during incubation at $37{ }^{\circ} \mathrm{C}$, mimicking the body temperature. This in vitro finding might suggest that newborns fed pasteurized human milk could be more susceptible to bacterial infections than those fed raw milk. Schanler et al. already reported more episodes of late-onset sepsis and NEC in premature infants fed pasteurized donor milk compared to those fed untreated mother's own milk [34].

Our study is limited by its small sample size and by the fact that all samples were refrigerated overnight prior to processing; this delay may have allowed lipolysis resulting in enhanced antimicrobial activity of both raw and pasteurized samples. Another weakness is that we did not include preterm formula milk in our assays. It would have been interesting to compare the antibacterial properties of both raw and pasteurized milk relative to formula milk instead of the tryptic soy broth control.

While the advantages of human milk for preterm infants have become more widely recognized over the last decade, little is known about the risks associated with feeding raw milk potentially contaminated with pathogens to preterm babies. In order to minimize exogenous contamination of expressed milk, stringent principles of hazard analysis and 
critical control points to ensure quality and safety should be established with priority [9]. Perhaps the most pressing task for future research is to answer several unresolved questions regarding bacteriological safety of mother's own milk for these immunologically vulnerable infants. For example, should a bacteriological screening of fresh milk be implemented prior to administration or is administration of pasteurized milk always preferable despite the considerable loss in antibacterial capacity [31]? Should we be cautious regarding the risks of feeding heat-treated milk containing bacteria potentially capable of producing heat stable enterotoxins (e.g., S. aureus)? Does feeding processed, sterile human milk in the first weeks of life influence the development of the intestinal microbiota of the preterm infant?

As the total antimicrobial protection provided by human milk appears to be far more than can be elucidated by examining protective factors individually, we believe that the impact of pasteurization of mother's own milk on the incidence of infection in premature infants can only be clarified through prospective trials.

Conflict of interest The authors declare that they have no conflict of interest.

\section{References}

1. Akinbi H, Meinzen-Derr J, Auer C, Ma Y, Pullum D, Kusano R, Reszka KJ, Zimmerly K (2010) Alterations in the host defense properties of human milk following prolonged storage or pasteurization. J Pediatr Gastroenterol Nutr 51:347-352

2. Araujo ED, Gonçalves AK, Cornetta Mda C, Cunha H, Cardoso ML, Morais SS, Giraldo PC (2005) Evaluation of the secretory immunoglobulin A levels in the colostrum and milk of mothers of term and preterm newborns. Braz J Infect Dis 9:357-362

3. Bertino E, Giuliani F, Occhi L, Coscia A, Tonetto P, Marchino F, Fabris C (2009) Benefits of donor human milk for preterm infants: current evidence. Early Hum Dev 85:S9-S10

4. Björksten B, Burman LG, De Chateau P, Fredrikzon B, Gothefors L, Hernell O (1980) Collecting and banking human milk: to heat or not to heat? BMJ 281:765-769

5. Carey AJ, Long SS (2010) Staphylococcus aureus: a continuously evolving and formidable pathogen in the neonatal intensive care unit. Clin Perinatol 37:535-546

6. Castellote C, Casillas R, Ramírez-Santana C, Pérez-Cana FJ, Castell M, Moretones MG et al (2011) Premature delivery influences the immunological composition of colostrum, transitional and mature human milk. J Nutr 141:1181-1187

7. Clare DA, Catignani GL, Swaisgood HE (2003) Biodefense properties of milk: the role of antimicrobial proteins and peptides. Curr Pharm Des 9:1239-1255

8. Cossey V, Jeurissen A, Bossuyt X, Schuermans A (2009) Effect of pasteurisation on the mannose-binding lectin activity and the concentration of soluble CD14 in human milk. J Hosp Infect 73:96-97

9. Cossey V, Jeurissen A, Thelissen M, Vanhole C, Schuermans A (2011) Expressed breast milk on a neonatal unit: a hazard analysis and critical control points approach. Am J Infect Control 39:832-838
10. Donowitz LW, Marsik FJ, Fisher A, Wenzel RP (1981) Contaminated breast milk: a source of Klebsiella bacteremia in a newborn intensive care unit. Rev Infect Dis 3:716-720

11. Eidelman AI, Schanler RJ (2012) American Academy of Pediatrics. Breastfeeding and the use of human milk. Pediatr 129:e827-e841

12. Ewaschuk JB, Unger S, Harvey S, O'Connor DL, Field CJ (2011) Effect of pasteurization on immune components of milk: implications for feeding preterm infants. Appl Physiol Nutr Metab 36:175-182

13. Gransden WR, Webster M, French GL, Phillips I (1986) An outbreak of Serratia marcescens transmitted by contaminated breast pumps in a special care unit. J Hosp Infect 7:149-154

14. Gras-Le Guen C, Lepelletier D, Debillon T (2003) Contamination of a milk bank pasteuriser causing a Pseudomonas aeruginosa outbreak in a neonatal intensive care unit. Arch Dis Child Fetal Neonatal Ed 88:F434-F435

15. Hartmann BT, Pang WW, Keil AD, Hartmann PE, Simmer K (2007) Best practice guidelines for the operation of a donor human milk bank in an Australian NICU. Early Hum Dev 83:667-673

16. Heikkilä MP, Saris PEJ (2003) Inhibition of Staphylococcus aureus by the commensal bacteria of human milk. J Appl Microbiol 95:471-478

17. Hodge D, Puntis JW (2000) The use of expressed breast milk for the premature newborn. Clin Nutr 19:75-77

18. Human Milk Banking Association of North America (2005) Guidelines for the establishment and operation of a human milk bank. HMBANA, Sandwich

19. Hylander MA, Strobino DM, Dhanireddy R (1998) Human milk feedings and infection among very low birth weight infants. Pediatr 102:38-43

20. Israel-Ballard K, Coutsoudis A, Chantry AW, Sturm AW, Karim F, Sibek AB (2006) Bacterial safety of flash-heated and unheated expressed breast milk during storage. J Trop Pediatr 52:399-405

21. Koenig A, de Albuquerque Diniz EM, Barbosa SF, Vaz FA (2005) Immunologic factors in human milk: the effects of gestational age and pasteurization. J Hum Lact 21:439-443

22. Kurath S, Halwachs-Baumann G, Müller W, Resch B (2010) Transmission of cytomegalovirus via breast milk to the prematurely born infant: a systematic review. Clin Microbiol Infect 16:1172-1178

23. Law B, Urias B, Lertzman J, Robson D, Romance L (1989) Is ingestion of milk-associated bacteria by premature infants fed by human milk controlled by routine bacteriologic screening. J Clin Microbiol 27:1560-1566

24. Lawrence RM, Pane CA (2007) Human breast milk: current concepts of immunology and infectious diseases. Curr Probl Pediatr Adolesc Health Care 37:7-36

25. Lucas A, Cole TJ (1990) Breast milk and neonatal necrotising enterocolitis. Lancet 336:1519-1523

26. Menon MP, Sobel J, Tauxe RV (2007) Pasteurization of banked human breast milk. Pediatr Infect Dis J 26:277-278

27. National Institute for Health and Clinical Excellence (2010) Donor breast milk bank. The operation of Donor Breast Milk Bank Services. Clinical Guideline CG93. At: http://www.nice.org.uk/ CG93. Accessed April 12, 2012

28. Ng DK, Lee SYR, Leung LCK, Wong SF, Ho JCS (2004) Bacteriological screening of expressed breast milk revealed a high rate of bacterial contamination in Chinese women. J Hosp Infect 58:146-150

29. Ogundele MO (2000) Techniques for the storage of human breast milk: implications for anti-microbial functions and safety of stored milk. Eur J Pediatr 159:793-797

30. Ogundele MO (2002) Effects of storage on the physicochemical and antibacterial properties of human milk. Br J Biomed Sci 59:205-211

31. Omarsdottir S, Casper C, Akerman A, Polberger S, Vanpée M (2008) Breast milk handling routines for preterm infants in Sweden: a national cross-sectional study. Breastfeed Med 3:165-170

32. Quigley M, Henderson G, Anthony M (2007) Formula milk versus donor breast milk for feeding preterm or low birth weight infants. Cochrane Database Syst Rev CD002971 
33. Schanler R, Shulman RJ, Lau C (1999) Feeding strategies for premature infants: beneficial outcomes of feeding fortified human milk versus preterm formula. Pediatr 103:1150-1157

34. Schanler RJ, Lau C, Hurst NM, Smith EO (2005) Randomized trial of donor human milk versus preterm formula as substitutes for mothers' own milk in the feeding of extremely premature infants. Pediatr 116:400-406

35. Silvestre D, Ruiz P, Martinez-Costa C, Plaza A, Lopez MC (2008) Effect of pasteurization on the bactericidal capacity of human milk. J Hum Lact 24:371-376

36. Stiver H, Albritton W, Clark J, Friesen P, White FM (1977) Nosocomial colonization and infection due to E. coli O125:K70 epidemiologically linked to expressed breast-milk feedings. Can J Public Health 68:479-482
37. Stoll BJ, Hansen NI, Sanchez PJ, Faix RG, Poindexter BB, Van Meurs KP, Bizzarro MJ, Goldberg RN, Frantz ID, Hale EC et al (2011) Early onset neonatal sepsis: the burden of group B streptococcal and E. coli disease continues. Pediatrics 217:817-826

38. Tully DB, Jones F, Tully MR (2001) Donor milk: what's in it and what's not. J Hum Lact 17:152-155

39. Vergnano S, Menson E, Smith Z, Kennea N, Embleton N, Clarke P, Watts T, Health PT (2011) Characteristics of invasive Staphylococcus aureus in United Kingdom neonatal units. Pediatr Infect Dis J 30:850-854

40. Vohr BR, Poindexter BB, Dusick AM, McKinley LT, Wright LL, Langer JC, Poole WK (2006) Beneficial effects of breast milk in the neonatal intensive care unit on the developmental outcome of extremely low birth weight infants at 18 months of age. Pediatr 118:115-123 